\title{
Acceleration of Electrons in a Self-Modulated Laser Wakefield
}

\author{
S.-Y. Chen, M. Krishnan, A. Maksimchuk and D. Umstadter
}

Center for Ultrafast Optical Srience, University of Michigan, Ann Arbor. MI 48109

\begin{abstract}
Acceleration of electrons in a self-modulated laser-wakeficld is investigated. The generated electron beam is oberved to have a multi-component beam profile and its cucrgy distribution undergoes discrete transitions as the conditions are variod. These features can be explained by simple simulations of electron propagation in a 3-D plasma wave.
\end{abstract}

Understanding the dynamies of clectron acceleration in an electron plasma wave is important for developing plasma-based electron accelerators [1]. Of the several methods for driving large-amplitude plasma waves, the laser wakefield accelerator (LWFA) and the self-modulated LWFA, have recently received considerable attention because of the reduction in size of terawatt class laser systems [2]. In the LWFA. an electron plasma wave is driven resonantly by a short laser pulse, and an additional injection mechanism is required [3.4]. In the self-modulated LWFA, an electron plasma wave is excited by a rclatively long laser pulse undergoing stimulated Raman forward scattering instability [5], and the injection of electrons is achieved by trapping hot background electrons which are preheated by other processes such as Raman backscattering and sidescattering instabilities $[6-8]$.

Several groups observed the generation of $\mathrm{MCV}$ electrons from the self-modulated LWFA [7-11]. A two-temperature distribution was reported in the electron energy spectrum [11]. R. Wagner et al. [10] observed that the generated electron beam has a multi-component beam profile, and that the temperature of the electrons in the low energy range undergoes abrupt change, coinciding with the onset of the extention of the laser channel by self-channeling of the laser pulse, when the laser power or plasma density is varied. Several 1-D and 2-D simulations [6,12-14] have also been done to study the electron beam characteristics of a self-modulated LWFA, in addition to the 1-D theoretical analysis $[15,16]$. However, no explanation were given for these experimental observations. In this Letter, we reported the observation of multi-component electron-beam profiles and discrete changes in the slope of electron energy distribution. These phenomena show more complicated behaviors compared to those reported before [10]. mainly due to the ability to reach higher laser intensity and plasma density. Most importantly, by using a

CP472, Advanced Accelerator Concepts: Eighth Workshop,

edited by W. Lawson, C. Bellamy, and D. Brosius

(C) 1999 The American Institute of Physics 1-56396-889-4/99/\$15.00 


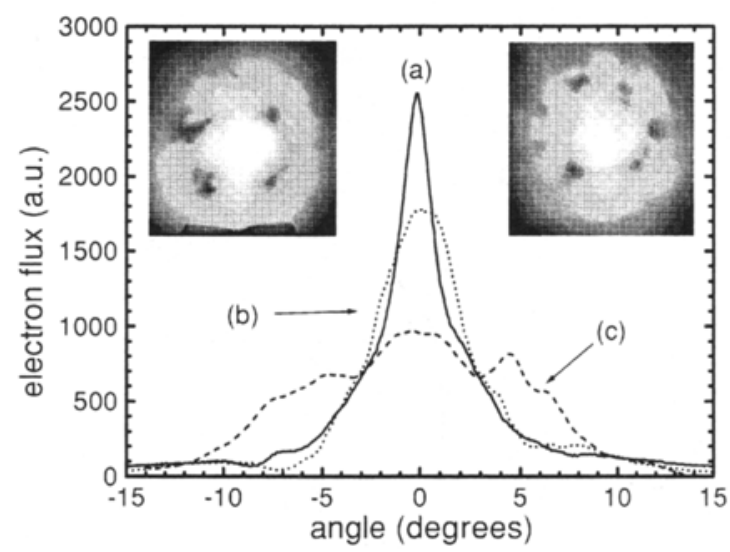

FIGURE 1. Lineouts of the electron beam profiles for differnt peak laser powers and plasma densities: (a) $2.9 \mathrm{TW}, 3.4 \times 10^{19} \mathrm{~cm}^{-3}$, (b) $3.5 \mathrm{TW}, 6.2 \times 10^{19} \mathrm{~cm}^{-3}$. and (c) $0.6 \mathrm{TW}, 2.3 \times 10^{19}$ $\mathrm{cm}^{-3}$. Inset on the left side shows the electron beam profile at $3.5 \mathrm{TW}$ and $6.2 \times 10^{19} \mathrm{~cm}^{-3}$. Inset on the right side shows the electron beam profile at $2.0 \mathrm{TW}$ and $2.3 \times 10^{19} \mathrm{~cm}^{-3}$.

simple 3-D simulation, we are able to explain these phenomena for the first time.

The experiment was done by using a laser system that produced 400 -fs laser pulses at $1.053-\mu \mathrm{m}$ with a maximum peak power of $4 \mathrm{TW}$. The 50-mm-diameter laser beam was focused with an $\mathrm{f} / 3.3$ parabolic mirror onto the front edge of a supersonic helium gas jet. The focal spot is a $7 \mu \mathrm{m}$ FWHM Gaussian spot, which contains $60 \%$ of the total energy, and a large dim spot (100 $\mu \mathrm{m}$ FWHM). The helium gas was fully ionized by the foot of the laser pulse. At the laser power of $\geq 2 \mathrm{TW}$ and the plasma density of $\geq 2 \times 10^{19} \mathrm{~cm}^{-3}$, the laser pulse undergoes relativistic-ponderomotive self-channeling $[10,17]$, and the laser channel extends to $750 \mu \mathrm{m}$ long, the length of the gas jet. The length of the laser channel was monitored by side imaging of Thomson scattering of the laser pulse in the plasma.

The generated electron beam can be characterized by its energy distribution (the longitudinal emittance) and beam divergence (the transverse emittance). The electron energy spectrum in the low energy range $(<8 \mathrm{MeV})$ was measured using a dipole permanent magnet with a LANEX scintillating screen imaged by a CCD camera as the detector. Higher-energy electron energy spectrum was obtained by using a dipole electromagnet and a multi-wire proportional chamber (MWPC). The electron beam profile was measured using a LANEX screen imaged by a CCD camera at $16 \mathrm{~cm}$ away from the gas jet. Since the source size of the generated electron beam is small, $\sim 10 \mu \mathrm{m}$ (determined by the diameter of the laser channel). the electron beam profile on the LANEX is actually a measurement of the electron beam divergence (angular pattern).

The electron beam profile (angular pattern) is observed to contain scveral concentric Gaussian-like-profile beams, and the number of the beam components depends on the laser power and plasma density. At the plasma density of $2.3-6.2 \times 10^{19}$ 


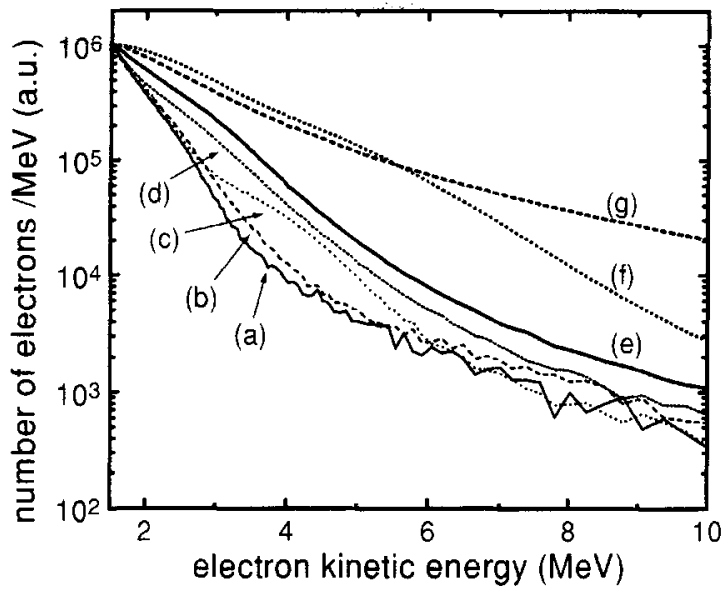

FIGURE 2. Electron energy spectra for different peak laser powers and plasma densities: (a) $2.6 \mathrm{TW}, 3.4 \times 10^{19} \mathrm{~cm}^{-3}$, (b) $2.9 \mathrm{TW}, 3.5 \times 10^{19} \mathrm{~cm}^{-3}$, (c) $3.3 \mathrm{TW}, 4.8 \times 10^{19} \mathrm{~cm}^{-3}$ : (d) 3.9 TW, $4.8 \times 10^{19} \mathrm{~cm}^{-3}$, (e) $1.7 \mathrm{TW}, 6.2 \times 10^{19} \mathrm{~cm}^{-3}$, (f) $2.7 \mathrm{TH}, 6.2 \times 10^{19} \mathrm{~cm}^{-3}$, and (g) 3.5 TW. $6.2 \times 10^{19} \mathrm{~cm}^{-3}$.

$\mathrm{cm}^{-3}$, only one beam component ( $\sim 15^{\circ}$ FWHM) exists in the electron beam for 0.6-TW laser power. For the laser power larger than $1 \mathrm{TW}$, gencrally two beam components were observed. which have the divergence of $15^{\circ}$ and $7.5^{\circ}$ FWHM, respectively. Lnder the condition of $2.0-3.5 \mathrm{TW}$ laser power and $2.3-3.4 \times 10^{19}$ $\mathrm{cm}^{-3}$ plasma density, a third beam component was observed and its divergence varies from $1.2^{\circ}$ to $2.5^{\circ}$. The electron flux of this third component can be as high as 10 times that of the second component. Figure 1 shows the lineouts of the electron beam profiles at three different conditions, corresponding to the cases of one-, two-, and three- component beam. Furthermore, when the second beam component shows up, there are usually some holes appearing in the first (widest) beam component. These holes form regular patterns, such as $\mathrm{TEM}_{10}$. TEM $\mathrm{T}_{11}$, and $\mathrm{TEM} \mathrm{M}_{12}$ modes. as shown in the insets of Fig. 1.

Figure 2 shows the normalized electron energy spectra in the low energy range for different laser powers and plasma densities. The spectra are found to have Maxwellian-like distributions, i.e., $\exp (-\alpha \gamma)$, where $\gamma$ is the relativistic factor of the electron energy and $\alpha$ is a fitting parameter $((511 \mathrm{keV}) / \alpha$ is the temperature $)$. The slope, $\alpha$, of the spectrum is found to change discretely with varying the laser power and plasma density. For instance, at a fixed plasma density, the slope stays the same with increasing laser power until a certain laser power is reached. Then the slope $\alpha$ changes to a lower value, and stays the same with further increase of laser power until the next jump. The same behavior occurs for varying plasma density at a fixed laser power. Three $a$ values (two jumps) were observed in this experiment: 1.0, 0.6, (0.3. The abrupt jump of the energy slope and the emergence of the second or third component in the electron beam profile do not have direct link 


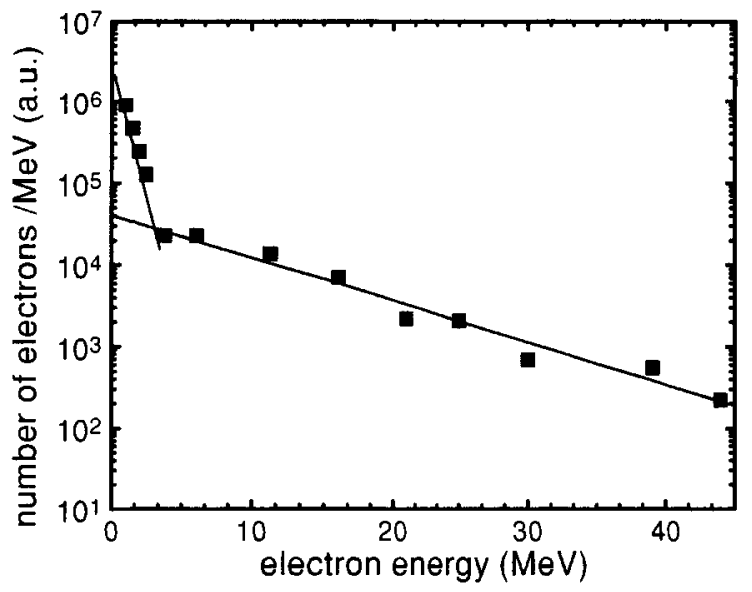

FIGURE 3. Electron energy spectrum for 3-TW peak laser power and $3 \times 10^{19}-\mathrm{cm}^{-3}$ plasma density.

with the occurrence of laser self-channcling. Another important obscrvation in this experiment is the two-temperature distribution in the electron energy spectrum. As shown in Fig. 3, which is obtained using high-energy electron spectrometer, the slope of electron energy distribution in the low energy range $(\leq 5 \mathrm{MeV})$ is steep, and the slope in the high energy range is much less stecp (almost flat).

To understand the physical origin of these phenomena, we run a simple 3-D particle simulation code and compare the results with the experimental observations. In this simulation, we inject monoenergetic electrons (with longitudinal kinetic energy $T_{e z}$ ) into presumed longitudinal and transverse electric fields of an electron plasma wave. The magnetic field is neglected in this simulation and the transverse electric field is derived from the longitudinal field by $\partial E_{r} / \partial z=\partial E_{z} / \partial r$ (results from the Maxwell's equations with B equaling to zero or a constant). The electric field assumed is

$$
\begin{aligned}
\vec{E}(r, \phi, z)= & \widehat{z} E_{0} \exp \left(-r^{2} / r_{0}^{2}\right) \cos \left(k_{p} z-\omega_{p} t\right) \\
& +\widehat{r} E_{0} k_{p}^{-1}\left(-2 r / r_{0}^{2}\right) \operatorname{cxp}\left(-r^{2} / r_{0}^{2}\right) \\
& \cdot \cos \left(k_{p} z-\omega_{p} t-\pi / 2\right) .
\end{aligned}
$$

, where $r_{0}$ is the radius of the plasma wave, $E_{0}$ is the peak longitudinal electric field, $k_{p}$ is the wave number of the plasma wave, and $\omega_{p}$ is the plasma frequency. The key feature in a self-modulated LWFA with self-trapping is that the electrons are injected everywhere along the channel of the plasma wave (determined by the lascr channel). 2000 clectrons are injected into a region of $r_{0}(\mathrm{x}) \times r_{0}(\mathrm{y}) \times \lambda_{p}$ (z) in one plasma period (one bucket). The momentum of each electron is saved whenever $t=n \cdot t_{p}$, where $t$ is the time since the injection, $t_{p}$ is the plasma period, and $n$ is an integer. The final result is the summation of all electrons saved, which is equivalent to injecting electrons uniformly over the entire channel. 

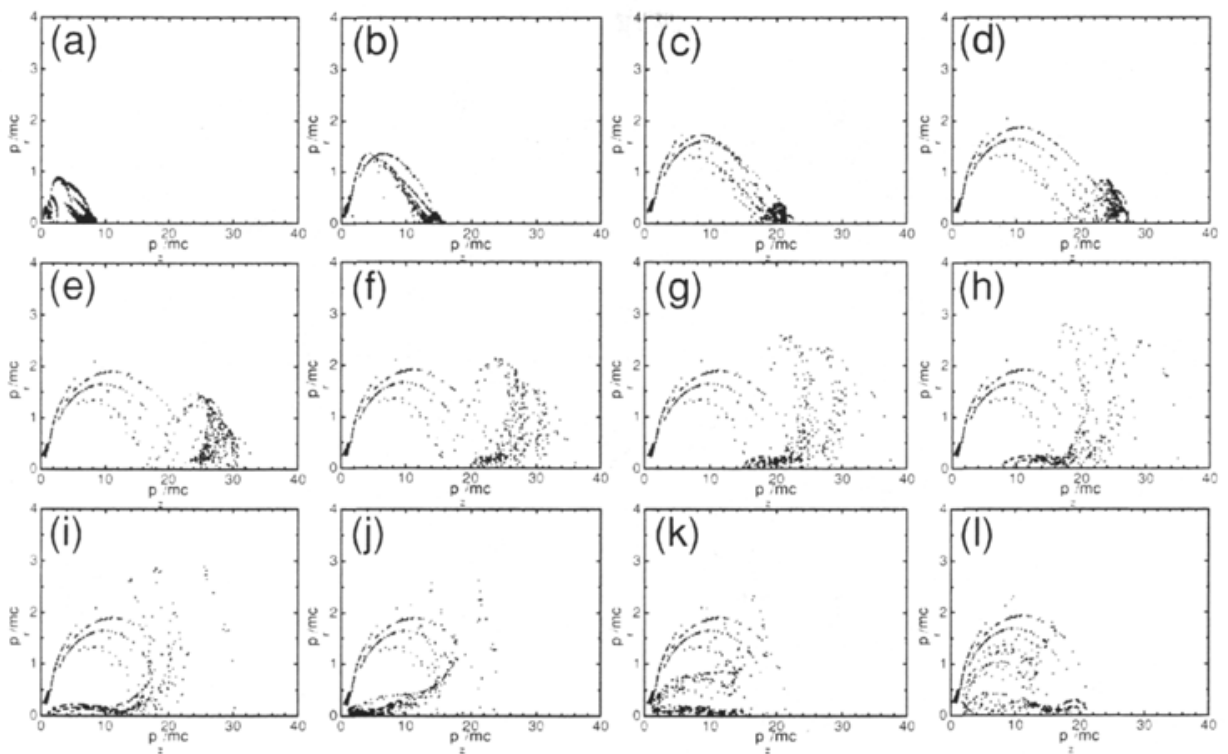

FIGURE 4. Simulational result of momemtum distribution of electrons injected in one plasma period after propagating different distances for $\epsilon=0.3, r_{0}=5 \mu \mathrm{m}, \omega_{p}=3.4 \times 10^{14} \mathrm{rad} / \mathrm{s}$, and $T_{\rho z}=200 \mathrm{keV}:$ (a) 22 , (b) 44, (c) 65, (d) 87, (e) 109, (f) 131, (g) 152 , (h) 174 . (i) 196. (j) 218, (k) 240 , and (l) $261 \mu \mathrm{m}$.

Figure 4 shows the evolution of momentum distribution of clectrons injected in the first plasma wave bucket for $E_{0} / E_{b}=0.3, r_{0}=\tilde{5} \mu \mathrm{m}, \omega_{p}=3.4 \times 10^{14} \mathrm{rad} / \mathrm{s}$, and $T_{e z}=200 \mathrm{keV}$, where $E_{b}=e \omega_{p} v_{p} / c$ is the nomrelativistic cold wavebreaking limit. After the injection, electrons that are not trapped (inside the separatrix $[1,16]$ ) are expelled by the transverse field to a contour defined by $\left(p_{r} / m c\right)^{2} / 2\left(p_{z} / m c\right)=$ $\epsilon\left(=E / E_{b}\right)$, where $p_{r}$ is the transverse momentum. The trapped electrons are mainly confined near $p_{r}=0$ and move toward higher $p_{z}$ (higher energy) with time. When they reach the maximum energy (the upper limit of the separatrix) after propagating one electron-detuning-length, $L_{d} \sim \gamma_{p}^{2} \lambda_{p}$, where $\gamma_{p}$ is the relativistic factor of phase velocity of the plasma wave, the electrons turn back and move toward the decreasing $p_{z}$ direction (lower energy). After the electrons reach the lower limit of the separatrix (the trapping threshold), they turn again and move toward higher $p_{z}$, and so on. While the trapped electrons move in an oscillatory trajectory inside the separatrix, they also drag a tail which spreads to the region confined by the contour, as a result of the transverse defocusing field of the plasma wave. As a result, less and less electrons are confined (guided by the transverse focusing ficld) in the region near $p_{r}=0$ (also the region near $r=0$ ), as they oscillate inside the separatrix. The time it takes for all the electrons to lose their confinement increases with increasing plasma wave amplitude. For example, for 

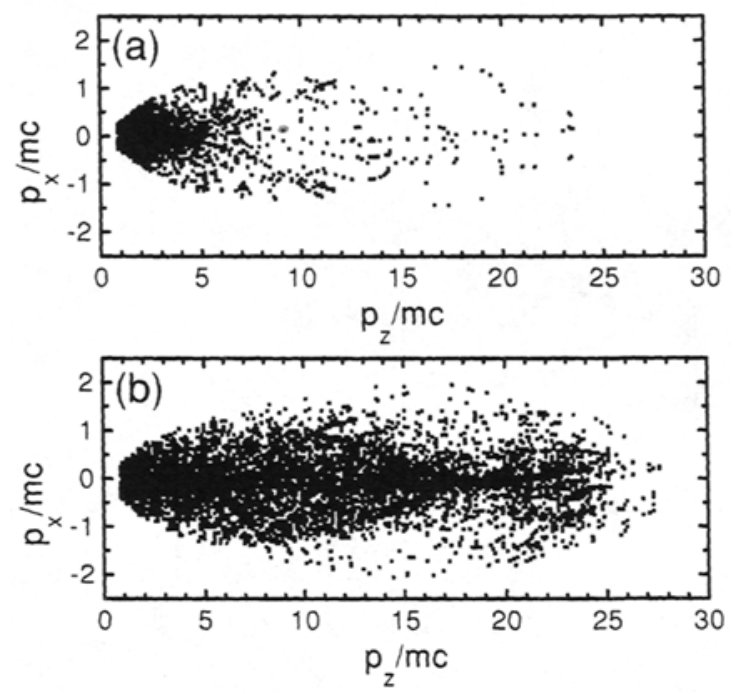

FIGURE 5. Simulational result of momentum distribution of clectrons injected orer the entire $400-\mu \mathrm{m}-\mathrm{long}$ channel for different field amplitudes at $r_{0}=5 \mu \mathrm{m}, \omega_{p}=3.4 \times 10^{14} \mathrm{rad} / \mathrm{s}$, and $T_{e:}=200 \mathrm{keV}:$ (a) $\epsilon=0.15$, and (b) $\epsilon=0.3$.

the parameters used in Fig. 4. the confinement time of the clectrons in the plasma wave with $\epsilon=0.15$. 0.2 and 0.3 is about one-fifth, twice and four times electron detuning length, respectively. The contour observed in the simulation results from the conservation of canonical momentum for the acceleration of an electron, which is at rest initially, by a plasma wave. This contour line is identical to the $p_{r}$ $p_{z}$ relation of the electrons accelerated by laser ponderomotive force (direct laser acceleration) when $\epsilon=1$ is used. Therefore, the appearance of electrons that satisfying $\left(p_{r} / m c\right)^{2} / 2\left(p_{z} / m c\right)=1$ in laser-plasma intcraction does not guarantee that it is the result of direct laser acceleration. It can come from the wavebreaking of plasma waves excited through Raman instability or resonant absorption.

The momentum distribution of the electrons injected over the entire channel for a channel length of $400 \mu \mathrm{m}$ are shown in Fig. 5. Generally, two groups of accelerated electrons are produced by the plasma wave, one is in the whole region confined by the contour, and another is in the region near $p_{r}=0$. These two groups of electrons results in the first and second component of the clectron beam profile observed in the experiment. That is, the first beam component (with larger divergence) results from electrons expelled by the transverse field before they exit the channel, and the socond beam component (with smaller divergence) results from electrous that are still confined in the channel transwersely when they exit the channcl. A third component is also observed in certain conditions in which the length of the channel is less than one clectron detuning length. Figure 6 shows the clectron beam profiles for several cases. As can be seen, the ratio between the divcrgences of different beam 


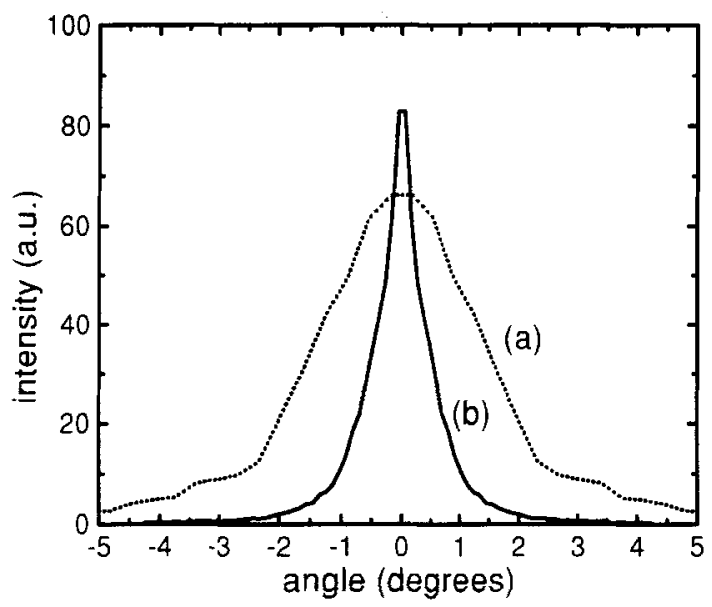

FIGURE 6. Simulational result of angular profile of the electron bean for different field amplitudes at $r_{0}=5 \mu \mathrm{m}, \omega_{p}=3.4 \times 10^{14} \mathrm{rad} / \mathrm{s}, T_{e:}=200 \mathrm{keV}$, and $L=400 \mu \mathrm{m}$ : (a) $\epsilon=0.3$, and (b) $\epsilon=0.1 j$.

components and between the intensities of these components can be reproduced in the simulation. In fact, the ratio between the divergence of the first and second beam components are found to be roughly a constant $(\sim 2)$ in both the results of the simulation and the experiment. The absolute value of the divergence is lower in the simulation (about a factor of 5 ), compared to the experimental results. The fact. that the beam divergence is larger in the experiment can be explained by several reasons. The most importance factor is the transverse space charge effect occurring during the acceleration and after exiting the channel. This is not considered in our simulations. Other effects such as nonlinear plasma wave and possible errors in measuring the plasma wave amplitude will also affect the result.

The electron energy spectra obtained from the simulations show a Maxwellian distribution in the low energy range and a flat region in the high energy range (and a high energy cutoff), as shown in Fig. 7. This is consistent with the experimental results. Furthermore, such a two-temperature distribution also appears in the 1-D simulation, as shown in Fig. 7 by setting $r_{0} / \lambda_{p} \simeq 100$. The exponential distribution in the low energy range is found to be composed of the untrapped but accelerated electrons (those outside the separatrix), and the newly trapped electrons which are trapped at the end of the channel. The encrgy distribution of the trapped electrons injected in a single bucket, is a narrow band with the central energy sweeping up and down inside the separatrix as the electrons propagate down the channel, as seen in Fig. 4. In the case of the self-modulated LWFA discussed here, the electrons are injected over the entire channel, and thus the spectrum of the electrons is the summation of all these narrow bands, leading to a flat-topped distribution in the high energy range.

Figure 8 shows the electron energy spectra for different channel lengths at $\epsilon=0.3$, 


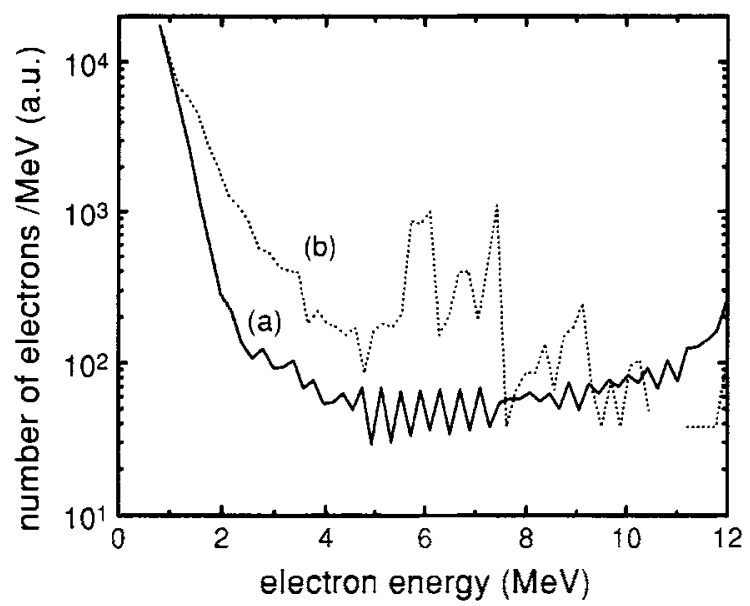

FIGURE 7. Simulational result of electron energy spectrum for different chammel radii at $\epsilon=0.15 . r_{0}=5 \mu \mathrm{m}, \omega_{p}=3.4 \times 10^{14} \mathrm{rad} / \mathrm{s} . T_{\epsilon:}=200 \mathrm{krV}$, and $L=400 \mu \mathrm{m}:$ (a) $r_{0}=500 \mu \mathrm{m}$, and (b) $r_{0}=5 \mu \mathrm{m}$.

$r_{0}=5 \mu \mathrm{m}, \omega_{p}=3.4 \times 10^{14} \mathrm{rad} / \mathrm{s}$, and $T_{e, z}=200 \mathrm{kcV}$. When the channel length is very short, the energy spectrum is an exponential distribution in the low energy range. With increasing the channcl length, while the slope of energy distribution in the low energy range maintains the same, the energy distribution in the high energy range becomes a flat-top with its maximum energy extending to higher energy. The flat-topped region reaches the upper limit of the separatrix after one electron-detuning-length, and then more electrons are added into the flat-topped region toward the lower energy direction with increasing channel length. After two electron-detuning-lengths, as the earliest-injected electrons travel back to the bottom (the low encrgy region) of the separatrix, the addition of these electrons to the low energy spectrum leads to a change in the slope of the exponential distribution. After the channel length is larger than two electron-detuning-lengths, the increase of channel length results in the increase of the electron number in the high energy region once again, and the slope of the energy distribution in the low energy range stays the same until the next jump which occurs at four electron-detuning-lengths.

The simulational results in Fig. 8 do not match well with the experimental results quantitatively. This is because that we consider only the clectrons injected at 200 $\mathrm{keV}$ energy. In reality, the injected electrons should have a continuous spectrum with a lot more clectrons at lower encrgy. These electrons with lower injection energy will have more electrons in the low-energy exponential distribution, which slope becomes steeper, and less electrons in the high-energy flat-top region, because less electrons are in the trapped (and confined) regions. Therefore, when injected with electrons with a continuous spectrum, we expect that the slope to be much stecper and the ratio between the numbers of electrons in the low energy range and the high energy range to be much larger. as in the experimental results. The discrete 


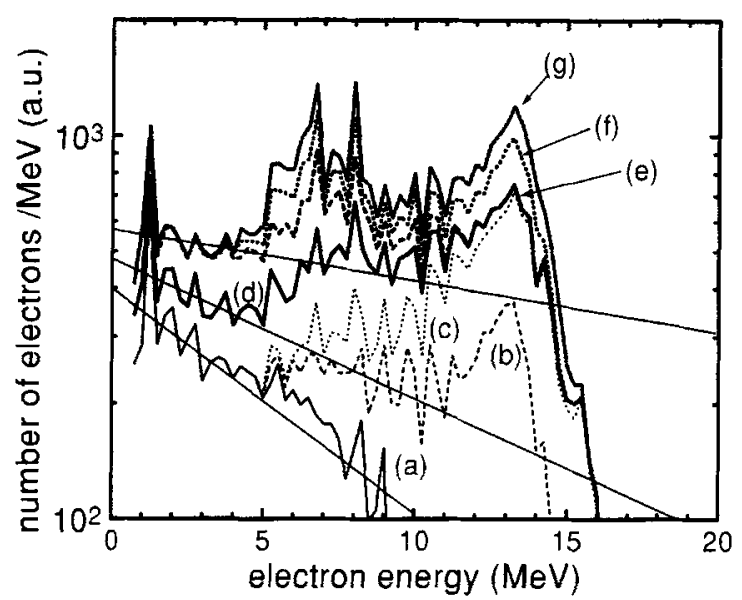

FIGURE 8. Simulational result of electron energy spectrum for clifferent channel lengths at $\epsilon=0.3, r_{0}=5 \mu \mathrm{ml}, \omega_{p}=3.4 \times 10^{14} \mathrm{rad} / \mathrm{s}$. and $T_{\epsilon:}=200 \mathrm{keV}:$ (a) 54 , (b) 109 . (c) 163 , (d) 218 , (c) 272 , (f) 327 .and $(\mathrm{g}) 381 \mu \mathrm{m}$.

jump of the slope of the low energy spectrum will still occur every twice electrondetuning-lengths, since the motion of the clectrons trapped in the separatrix are basically the same regardless of the injection energy. To compare this with the experimental results, we plotted the experimental data on a $\alpha-\left(L / 2 L_{d}\right)$ diagram, as shown in Fig. 9, in which $L$ is the channel length, and $L_{d}$ is determined from the plasma density. The results show jumps occurring when $L / 2 L_{d}$ equals to an integer. as expected from the simulations. Qualitatively, the increase in the channel length or the plasma density (decreasing $L_{d}$ ) change $L / 2 L_{d}$ to a larger value, and abrupt changes of the slope is expected to occur at the integers. For the cases in which the laser power is increased at a fixed plasma density and a long channel length (fixed $L / 2 L_{d}$ ), the jump of slope still happens because the confinement time of electrons depends on the plasma wave amplitudes. In these cases, $L$ should be replaced by the confinement length, which increases with increasing the amplitude of the plasma wave (with increasing the laser power or plasma density).

In conclusion, the characteristics of the electron beam generated from a selfmodulated LWFA are measured in the experiment, and the main features in the beam profile and the energy spectrum can be understood with the help of a simple 3-D particle simulation. Because of its simplicity, this simulation can not be used to explain the observed dark mode structures in the first beam component. There are at least two possibilities for such phenomena. The mode structure could be a result of the complicated transverse structure of the plasma wave induced by the nonlinearity of a large-amplitude plasma wave. It can also result from electron beam instabilities induced by magnetic fields, such as the Weibel instability [18] and the Kelvin-Helmholtz instability [19]. This work is supported by NSF PHY 972661 and NSF STC PHY 8920108. The author would like to thank E. Dodd, 


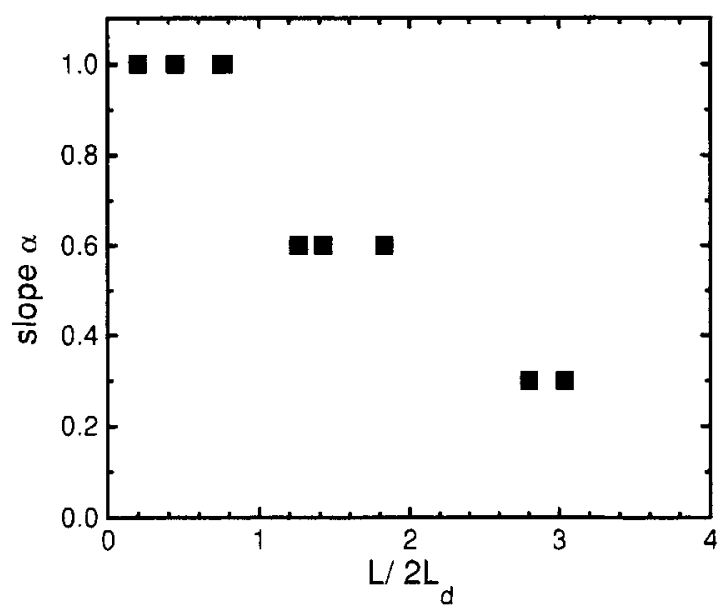

FIGURE 9. Slope of the electron energy spectrum in the low energy range as a function of $L / 2 L_{d}$.

J.-K. Kin, G. Mourou, and R. Wagner for their useful discussion.

\section{REFERENCES}

1. See references in E. Esarey et al., IEEE Trans. Plasma Sci. PS-24, 252 (1996).

2. P. Maine et al., IEEE J. Quantum Electron. 24, 398 (1988).

3. D. Umstadter, J. K. Kirn, and E. Dodd, Phys. Rev. Lett. 76, 2073 (1996).

4. E. Esarey et al., Phys. Rev. Lett. 79, 2682 (1997).

5. P. Sprangle et al., Phys. Rev. Lett. 69, 2200 (1992); T. M. Antonsen and P. Mora. Phys. Rev. Lett. 69, 2204 (1992); N. E. Andrecv ct al., JETP Lett., 55, 571 (1992).

6. P. Bertrand et al., Phys. Rev. E 49, 5656 (1994).

7. D. Umstadter et al., Science 273, 472 (1996).

8. C. I. Moore et al., Phys. Rev. Lett. 79, 3909 (1997).

9. A. Modena et al., Nature 377, 606 (1995).

10. R. Wagner et al., Phys. Rev. Lett. 78, 3125 (1997).

11. G. Malka et al., Phys. Rev. Lett. 79, 2053 (1997).

12. C. D. Decker, W. B. Mori, and T. Katsouleas, Phys. Rev. E 50, R3338 (1994).

13. N. E. Andreev, L. M. Gorbunov, and S. V. Kuznetsov, IEEE Trans. Plasma Sci. 24, 448 (1996).

14. K.-C. Tzeng et al., Phys. Rev. Lett. 79, 5258 (1997).

15. P. Mora and F. Amiranoff, J. Appl. Phys. 66, 3476 (1989).

16. E. Esarey and M. Pilloff Phys. Plasmas 2, 1432 (1995).

17. S.-Y. Chen et al., Phys. Rev. Lett. 80, 2610 (1998).

18. E. S. Weibel, Phys. Rev. Lett. 2, 83 (1959).

19. C. F. Driscoll and K. S. Fine, Phys. Fluids B 2, 1359 (1990). 